\title{
Multicast Routing in WDM Networks without Splitters
}

\author{
Dinh Danh Le, Miklós Molnár and Jérôme Palaysi
}

\begin{abstract}
Multicasting in WDM core networks is an efficient way to economize network resources for several multimedia applications. Due to their complexity and cost, multicast capable switches are rare in the proposed architectures. The paper investigates the multicast routing without splitters in directed (asymmetric) graphs. The objective is to minimize the number of used wavelengths and if there are several solutions, choose the lowest cost one. We show that the optimal solution is a set of light-trails. An efficient heuristic is proposed to minimize conflicts between the light-trails, and so to minimize the number of used wavelengths. The performance is compared to existing light-trail based heuristics. Our algorithm provides a good solution with a few wavelengths required and a low cost.
\end{abstract}

Keywords: WDM network, multicast routing, light-trail, wavelength minimization, heuristic

\section{INTRODUCTION}

All-optical networks are serious candidates to become high speed backbone networks with huge capacity. In optical routing, the messages are transmitted by light signal without electronic processing. Routes should satisfy the physical (optical) constraints in static connection based networks and also in the case of burst and packet switching.

Multicast communications are present in networks to efficiently perform data transmission from a source to several destinations. Usually, without physical constraints, multicast routes corresponds to trees in the topology graph. To perform multicast, there should be multicast capable nodes (splitters) at all the branching nodes of the tree. However, one of the most hard constraints for optical multicasting is the constraint on the availability of light splitters in the switches. In fact, splitters are expensive and the light power can be decreased considerably by splitting (inversely proportional with the number of outgoing ports [2]). This constraint prevents all-optical multicasting from employing splitters.

In our paper, we investigate an interesting question: how to perform multicast without splitters? Trivially, a set of lightpaths from the source to the destinations can be used as a solution, but this solution is expensive in term of wavelengths. Our objective is to perform multicasting without splitters and minimizing the number of used wavelengths. Solutions in bidirectional networks (where a wavelength is available in both directions between the connected switches) are known, but we investigate the arbitrarily directed case which is very practical. Even if the network is designed to be bidirectional, when

Manuscript submitted April 2013, revised May 28, 2013. This work is sponsored in part by VIED, Vietnam.

Dinh Danh Le, Miklós Molnár and Jérôme Palaysi are with the laboratory LIRMM, Université Montpellier 2, France (email: dinhdanh.le@lirmm.fr miklos.molnar@lirmm.fr, jerome.palaysi@lirmm.fr). some demands hold some of the resources of the network, the resulting network graph is now arbitrarily directed, therefore the routing for subsequent demands will be calculated on a digraph.

Due to its interest, WDM multicast routing has been investigated intensively in the literature and several propositions exist to adapt multicast routing algorithms to the optical constraints (cf. [9] for some basic algorithms and [11] for a survey). The minimization of the number of used wavelengths was investigated at first in [5] where the wavelengths are supposed to be unevenly distributed in the networks. The considered network is assumed to be equipped with splitters and wavelength converters. The multicasting is based on a tree: the objective is to construct a tree $T$ meeting optical constraints such that the number of wavelengths used to cover $T$ is minimized. The NP-hardness of the problem is proved, and an approximation algorithm has been proposed. An improved approximation can be found in [8].

The case of switching without splitters in symmetric networks has been discussed in [1]. The problem is to find a Multiple-Destination Minimum Cost Trail (MDMCT) that starts from a source and spans all the destinations with minimizing the total cost of the edges traversed. To ensure a feasible solution, a low-cost cross-connect architecture called Tap-and-Continue $(\mathrm{TaC})$ has been proposed to replace splitters. $\mathrm{TaC}$ cross-connects can tap a signal with small power at the local station and forward it to one of its output ports. Moreover, every link is assumed to be equipped with at least two fibers in order to support bidirectional transmission on the same link.

The authors proved that the MDMCT problem is NPhard and then developed a heuristic (called MDT) that finds a feasible trail in polynomial time. The algorithm has two steps. The first step is computing an approximated Steiner tree for a multicast request using the Minimum Cost Path Heuristic proposed in [7]. A trail is then computed based on the backtracking method following the tree.

The advantage of MDT heuristic is that it uses only one wavelength (and one transmitter) for each multicast request (and thus, the wavelength is minimized). However, because of multitude of round-trip traversing, a large number of links is required in both directions, hence the total cost and the diameter of the light-trail is always very high. To improve the total cost, it is necessary to reduce the round-trip traversing. Moreover, it is worth noting that, the source can inject the light signal by multiple transmitters independently. By taking this feature into account, one can considerably reduce the reversal arcs (that backtrack to the source), then the total cost and 
the diameter can also be reduced. This is the idea to make a modified version of MDT, called MMDT that is detailed in Section V.

In [3], Der-Rong Din posed the Minimal Cost Routing Problem which minimizes the cost under WDM symmetric networks using only TaC cross-connects. Unlike the approach of [1] that based on light-trail, the approach of Der-Rong Din is based on light-forest (a set of the light-trees [11]), rooted at the source and covering all the destinations. Besides, the source can inject the signal by multiple transmitters so that each light-tree can use a single wavelength. Furthermore, to produce a trade-off between the total cost of the light-forest and the number of wavelengths used, the author developed an objective function which combines the actual total cost of the light-forest and the cost for using wavelengths: $f=$ $\operatorname{cost}(F)+\alpha * n u m W L$, in which $F$ is the resultant lightforest, numWL is the number of wavelengths used, and $\alpha$ is a specific coefficient.

The author proposed two heuristic algorithms, namely Farthest-Greedy (FG) and Nearest-Greedy (NG). The two algorithms are based on the shortest path tree (SPT). The idea of these algorithms is: first construct the SPT from the source to the destinations, then keep one path for each subtree of the source, and finally reroute the other destinations that have not been reached (unreached destinations). The difference between the two algorithms is: FG keeps the farthest (in term of cost) destination routed by the computed shortest path, and chooses the farthest destination in the unreached set to reroute, whereas NDF keeps the nearest (minimal cost) destination and chooses the nearest destination in unreached set to reroute in the rerouting phase. The rerouting phase is performed by the shortest paths from the source or from the leaves of computed trees to each unreached destination, that do not share any nodes and edges with all the computed trees (each tree is computed in a different wavelength graph that is initialized by the original graph). When there is no possible path in the computed trees or the path exists but with larger cost than the path found in the new wavelength graph, the unreached destination is routed by the shortest path found in the new tree with a new wavelength. The author also gave the comparison between FG, NG and MDT by simulations, and the results show that FG is better than NG and MDT.

Most of the solutions proposed in the literature (excluding MDT) are based on simple routes in which cycles are not allowed. However, one can operate multicasting by non-simple routes which permit nodes to be visited several times, as long as the routes using the same wavelength are arc-disjoint. MDT in [1] gives a special structure which allow cycles, but with special cycles which are 2 -cycles ${ }^{1}$. In fact, one can construct structures that allow not only 2-cycles but also arbitrary cycles. These structures correspond to a hierarchy that was proposed in [6]. For multicast routing in WDM networks, the lighthierarchy concept has been proposed in [10]. A light-hierarchy is a hierarchy using a single wavelength.

In the paper, we show that the optimal route minimizing the number of wavelengths is a set of (non-simple) light-trails. The

${ }^{1}$ An $n$-cycle is a cycle with $n$ vertices. computation of the optimum in directed graphs is NP-hard. So, we propose some heuristic algorithms, which try to minimize the number of wavelengths, taking into account the availability of fibers in the network, with a low cost. We compare the performance of them with two previously proposed multicast routing algorithms.

The structure of the paper is the following. Section II formulates the problem. Several useful concepts and properties are given in Section III. Our heuristics are described in Section IV followed by the experimental results in Section V.

\section{Problem Formulation}

Let us suppose that nodes are equipped by $\mathrm{TaC}$ option and can be traversed by the same wavelength several times having different incoming and outgoing ports for each pass. So, lighttrails from the source to the destinations can perform the multicast (cf. [1]). There is no splitter in the nodes. Moreover, the topology graph $G=(V, A)$ is an arbitrary directed graph in which each arc represents a fiber between the pair of nodes. Each $\operatorname{arc} e \in A$ is associated with the a value $\operatorname{cost}(e)>0$. Let $s \in V$ the source and $D \subseteq V \backslash\{s\}$ the set of destinations. We suppose that the multicast is feasible, i.e., there is at least one directed path from the source to each destination. Different objectives for the multicast routing can be formulated as follows.

Routing using a minimum number of wavelengths $\left(P_{1}\right)$ : A multicast route colorable with the least number of wavelengths is required.

Minimum cost routing $\left(P_{2}\right)$ : The least cost multicast route is required.

In both cases (with the given constraints), the optimal solution is a set of light-trails routed at the source and covering all of the destinations. Notice that this set corresponds to a hierarchy obtained from a star (cf. [6] for the definition of a hierarchy). The solution of Problem $P_{1}$ can be composed from very long trails. The optimum of Problem $P_{2}$ can use a high number of wavelengths. Trade-off can be interesting.

Minimum cost multicast routing using a given number of wavelengths $\left(P_{3}\right)$ : A minimum cost multicast route colorable with the given number of wavelengths is required.

Length limited multicast route using a minimum number of wavelengths $\left(P_{4}\right)$ : A multicast route in which the longest trail is smaller than a given limit and colorable with the minimum number of wavelengths is required.

The solutions of the last two problems are also sets of lighttrails.

The mentioned routing problems are hard optimization problems. Problem $P_{1}$ corresponds to finding the solution with minimal set of colors. Problem $P_{2}$ is equivalent the Degree Constrained Directed Minimum Spanning Tree Problem in the distance graph of the problem.

In our study, we focus on the first problem but some results can be useful to solve Problem $P_{3}$ and Problem $P_{4}$. For the limited space, the reader is recommended to refer our research paper [4] for the proof of NP-hardness of $P_{1}$. 


\section{USEFUL DEFINITIONS AND PROPERTIES}

In order to describe our algorithm, some concepts should be given in the following.

- Directed shortest path tree (DSPT): A directed tree rooted at the multicast source covering all the destinations by shortest paths, in which each path is (one of) the shortest path(s) from the source to a destination. To compute DSPT, any shortest path algorithms, e.g., Dijkstra algorithm, can be employed.

- Conflict graph: A graph used to represent the conflicts among the trails. Formally, in our study, a conflict graph is $G_{C}=(T, E)$, in which $T$ is a set of nodes corresponding to the trails and $E$ is a set of edges such that $e=\{t, p\} \in E$ if and only if there is a conflict between trail $t$ and trail $p$, i.e., two trails share a common arc. In this study, we just consider conflicts such that shared arcs form the prefix of the concerned trails and this property is preserved during the algorithm. For this condition, each of connected components in the conflict graph corresponds to a subtree of the DSPT (Figure 1).

Property 1: Each connected component in the conflict graph composes a (conflict) clique ${ }^{2}$.

Trivially, if the shared arcs of conflicting trails are the prefix of the trails, then conflicts are transitive (if there is a conflict between $T_{1}$ and $T_{2}$ and between $T_{2}$ and $T_{3}$, then there is a conflict between $T_{1}$ and $T_{3}$ ). Indeed, all the trails in the same connected component share the first arc from the source. So the Property 1 follows.

Property 2: The number of colors needed to color all the nodes of a clique is equal to the number of nodes of that clique.

Obviously, there is a (conflict) edge between every pair of nodes in each clique. To avoid the conflict, the nodes must be colored with different colors. So the Property 2 follows.

Property 3: The number of wavelengths needed to perform the routing respecting the distinct wavelength constraint $^{3}$ in network fibers is equal to the number of nodes (the size) of the maximal clique in the conflict graph in our study case.

In deed, each clique corresponds to a subtree of the DSPT. These subtrees are arc-disjoint, so the corresponding trails in each clique do not share any arcs with the corresponding trails in the other ones. Thus, the minimal number of colors needed to color all the nodes in the conflict graph is equal to the size of the maximal clique, because we can use some colors that have been used in the maximal clique to re-color the other nodes in the other cliques. Moreover, to guarantee the distinct wavelength constraint, the number of colors needed in each clique is equal to the number of wavelengths needed to assign the corresponding trails in that clique. So this property holds.

\footnotetext{
${ }^{2} \mathrm{~A}$ clique of a graph is a complete subgraph of that graph

${ }^{3}$ Distinct wavelength constraint: Different light-paths or light-trees sharing the common link must be allocated distinct wavelengths [11].
}

Thus, the problem of minimizing the number of used wavelengths reduces to the problem of minimizing the number of nodes (trails) of the maximal clique in the conflict graph.

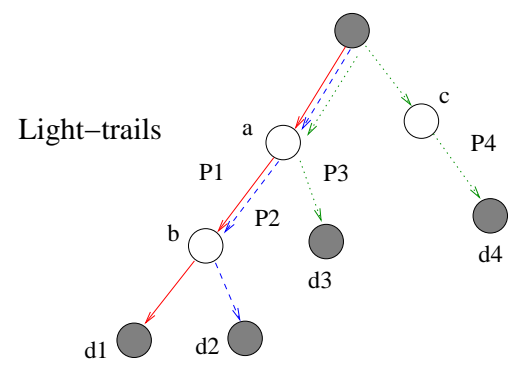

Conflict graph

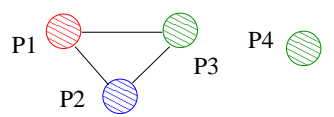

Fig. 1: Example of a DSPT and its conflict graph

Figure 1 illustrates a set of paths (trails) composing a DSPT for the multicast request $r=\left(s,\left\{d_{1}, d_{2}, d_{3}\right\}\right)$ and the corresponding conflict graph. In Figure 1, there are two cliques corresponding to two subtrees of the DSPT. The maximal clique is composed from the paths $T_{1}, T_{2}, T_{3}$ starting from the source to the destinations $d_{1}, d_{2}, d_{3}$, respectively. It needs three wavelengths to color the three trails. The other clique composed from only the path $T_{4}$ that can re-use one wavelength that were assigned for the maximal clique.

\section{Proposed Heuristics}

\section{A. Algorithm Framework}

The idea of the algorithm is to diminish the number of nodes (trails) in the maximal clique of the conflict graph until it cannot be reduced. Informally, the algorithm starts from a set of directed trails (at first, simple trails or paths in the DSPT). Then it tries to iteratively diminish the number of trails in the maximal clique, say $C_{\max }$. At each step, it chooses one trail from the maximal clique, say $T_{0}$, that can be replaced by another one. Some mechanism can be employed to select this trail. When the trail is selected, the algorithm looks for all the other trails and choose the one, say $T_{k}$, such that the terminal of it has the arc-disjoint shortest path to the first destination of the trail $T_{0}$. Then $T_{0}$ is replaced by the corresponding trail $T_{k}$, and the cardinality of the $C_{\max }$ is reduced by 1 . The algorithm iterates until the maximal clique cannot be reduced.

The framework of the algorithm consists of four main steps that can be described as follows:

\section{The Algorithm Framework}

Step 1: Compute a directed shortest path tree (DSPT) from the source $s$ covering all destinations. If there is no 
branching node in the DSPT, then DONE. Otherwise, do Step 2.

Step 2: Compute the conflict graph from the DSPT. Each conflict clique corresponds to a sub-tree rooted at the source of the DSPT.

Step 3: Repeat Step (3.1) to Step (3.4) in the following until the cardinality of the maximal clique $C_{\max }$ cannot be reduced (is equal to 1 for the best case).

Step 3.1: Find the maximal clique $C_{\max }{ }^{4}$.

Step 3.2: Choose a trail $T_{0}$ in $C_{\max }$ with some mechanism that is mentioned below. Calculate the terminal $l_{0}$ of $T_{0}$ and the first destination $f_{0}{ }^{5}$ of $T_{0}$. For example, in Figure 1 a), $C_{\max }$ is composed by the paths $T_{1}, T_{2}, T_{3}$; the selected trail $T_{0}$ is $T_{3}$, $l_{0}$ is node $d_{3}$ and $f_{0}$ is also $d_{3}$.

Step 3.3: For every terminal $l_{i}$ of the remaining trails $T_{i}$ in the set of trails (except $T_{0}$ ), compute the trail $T_{k}$ such that the path $\left(l_{k}, f_{0}\right)$ is arc-disjoint with all the current trails, and it is the shortest path among the paths $\left(l_{i}, f_{0}\right)$. If there is an arcdisjoint path from the source $s$ to $f_{0}$, then take the shorter one between $\left(l_{k}, f_{0}\right)$ and $\left(s, f_{0}\right)$. In Figure $1 \mathrm{a}), T_{k}$ is $T_{4}$, and $l_{k}$ is node $d_{4}$.

Step 3.4: Graft the path $\left(l_{k}, f_{0}\right)$ and the path $\left(f_{0}, l_{0}\right)$ to the trail $T_{k}$, set $l_{0}$ as the terminal of the new trail $T_{k}$, remove the trail $T_{0}$, reduce the cardinality of clique $C_{\max }$ by 1 . If the path $\left(s, f_{0}\right)$ exists and is selected, create a new trail $\left(s, l_{0}\right)$ and remove the trail $T_{0}$.

Step 4: Record the set of trails, the cardinality of clique $C_{\max }$ as the minimum number of wavelengths required. Employ the trail-wavelength-assignment (TWA) algorithm (described below) to assign wavelengths for the set of final trails.

\section{B. Trail-wavelength-assignment (TWA) algorithm}

The TWA algorithm mentioned in Step 4 works as follows. Let $k$ be the minimum number of wavelengths returned by the routing algorithm above, and $w_{1}, w_{2}, . ., w_{k}$ be the $k$ wavelengths reserved for the multicast request. With each wavelength $w_{i}, i=1, \ldots, k$, assign $w_{i}$ for every clique in the set of the remaining cliques, one trail for each clique. Repeat that until there is no trail in the set of remaining cliques.

\section{Two greedy heuristics}

Our algorithm can be developed to result in two heuristics. In step 3.2, two greedy mechanisms to select the first trail $T_{0}$ in the maximal clique $C_{\max }$, resulting in two heuristics of our algorithm, namely Farthest First (FF) if $T_{0}$ is the longest trail in term of cost among all the other trails in $C_{\max }$, i.e., the terminal $l_{0}$ of $T_{0}$ is the farthest terminal $l_{0}$ among the others

\footnotetext{
${ }^{4}$ To accelerate this step, $C_{i}$ is organized in a priority queue in which the priority value is the size of $C_{i}$, and only cliques $C_{i}$ with the size larger than 1 are pushed into the queue

${ }^{5} f_{0}$ is the first destination on the path from the nearest branching node of $l_{0}$ to $l_{0}$
}

in $C_{\text {max }}$; and Nearest First (NF) if $l_{0}$ is the nearest terminal in $C_{\max }$. The detail descriptions of the two heuristics and the complexity of them are given in our research paper [4].

\section{Illustration of the algorithm}

In order to demonstrate the algorithm, we use a network in Figure 2 in which the source is $s$, the destination set is $D=\{2,6,7,8,10,11,12,13\}$. Moreover, due to the limited space, and because FF and NF have the same principle, we just illustrate the heuristic FF in the Figure 3 below.

After the Step 1 and Step 2, the DSPT and the initial conflict graph are shown in Figure 3 (a). The maximal clique comprises three paths $T_{10}, T_{12}, T_{13}$, in which $T_{12}$ is the farthest one, so it is selected first. The first destination $f_{0}$ of $T_{12}$ is node 8 , the shortest arc-disjoint path computed is the path passing the nodes $\{10,5,8\}$. Thus $T_{12}$ is replaced by $T_{10}$, the new trail is $T_{12}^{\prime}$ (Figure $3(\mathrm{~b})$ ). Similarly, $T_{12}^{\prime}$ is then replaced by $T_{12}^{\prime \prime}$ in the next run (Figure 3 (c)). The final set of trails are shown in Figure $3(d)$.

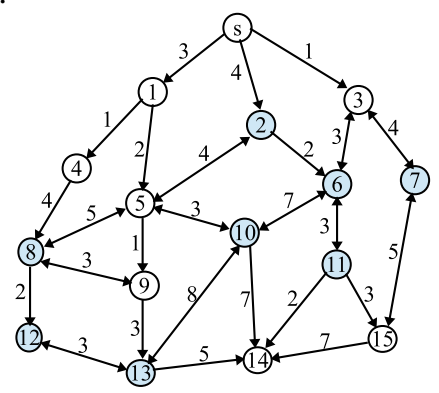

Fig. 2: The network to consider
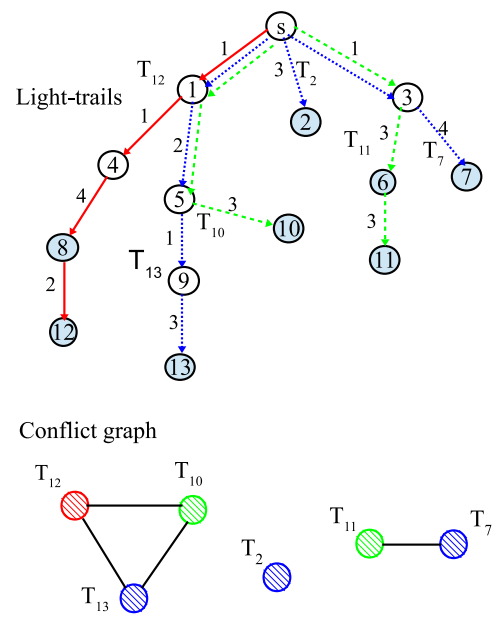

(a) The DSPT and the initial conflict graph

\section{EXPERIMENTAL RESULTS}

In this section, we show the performance of our algorithm and compare with the algorithms proposed in [1] (MDT) and [3] (Farthest Greedy and Nearest Greedy). In order to fairly compare with MDT in [1], the modified version of it is developed, namely Modified-MDT (MMDT for short) which is mentioned in the following. 


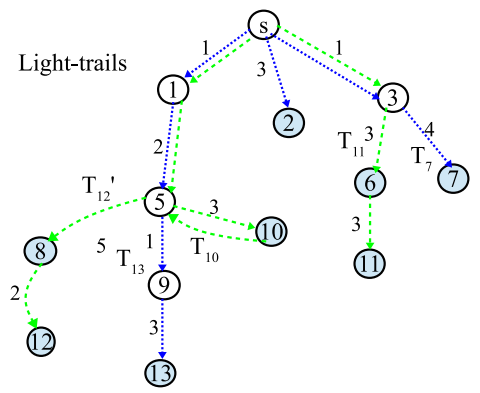

Conflict graph

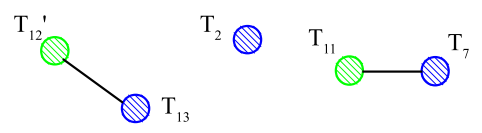

(b) Replace $T_{12}$ by $T_{12}^{\prime}$
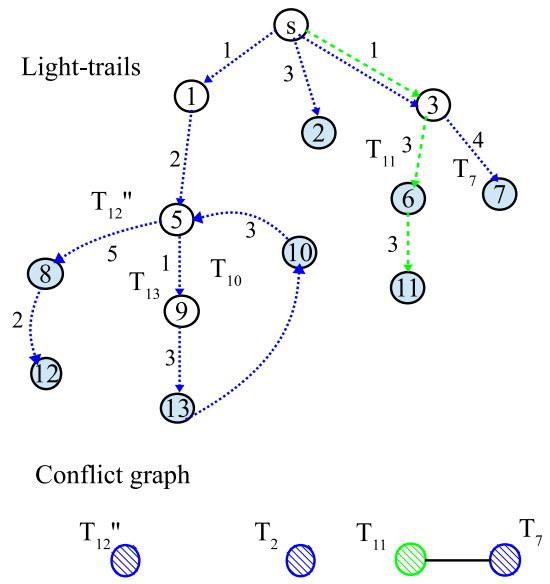

(c) Replace $T_{12}^{\prime}$ by $T_{12}^{\prime \prime}$

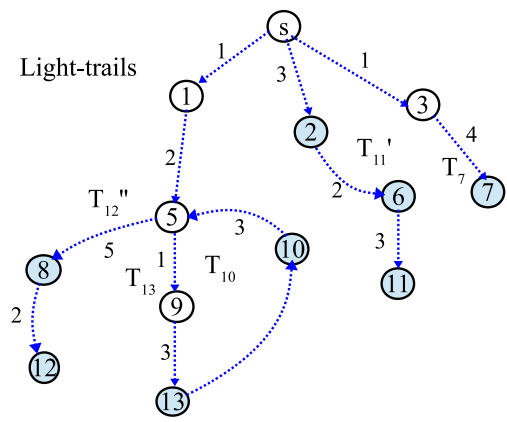

Conflict graph

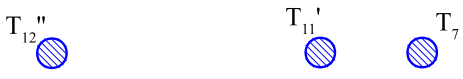

(d) Replace $T_{11}$ by $T_{11}^{\prime}$

Fig. 3: Illustration of the Farthest First heuristic

\section{A. MDT and MMDT}

As mentioned in Section II, the MDT algorithm has two steps. The first step is to compute an approximated Steiner tree (AST) for a multicast request using the Minimum Cost Path Heuristic (MCPH) proposed in [7]. In the second step, a trail is computed based on the backtracking method following the AST. The backtracking phase starts from the root of the tree, and recursively repeats at each non-leaf node in the tree, say, the current node. In the downstream direction, the algorithm tries to include all the downstream links between the current node and all its children destinations. Backtracking is required when a leaf node is reached and there are still some destination nodes not yet visited.

However, the total cost and the diameter of the MDT trail are high because of multitude of round-trip traversing. Moreover, it is worth noting that, the source can inject the light signal by multiple transmitters on the same wavelength independently. By taking advantage of this feature, we developed the algorithm MMDT by modifying MDT in the backtracking phase, in such a way that it can eliminate the round-trip traversing the source while using only one wavelength.

The MMDT works as follows. First, it generates an AST using the MCPH just like the way of MDT. Then the backtracking method to each subtree of the AST (the nodes 1,2 and 3 in Figure 4) is evoked, with a greedy sequence such that the trails growing to the nearest branch first (in term of cost of the branch). Consequently, there is no reversal arcs needed in the farthest branch for each sub-tree. Accordingly, the result is the set of trails rooted at the source, covering all the destinations with only one wavelength, but with multiple transmitters, one transmitter for each trail. Obviously, the diameter and the total cost of the resultant trials are less than those resulted by MDT.

To demonstrate MDT and MMDT, we use the same topology as the one shown in Figure 2 with a few changes: all the links are now bidirectional and the destination set is $D^{\prime}=\{2,5,6,7,8,9,10,11,12,13\}$. Figure 4 (a) demonstrates the computation of the multiple-destination trail according to the MDT algorithm and Figure 4 (b) demonstrates the MMDT for the same request $r=\left(s, D^{\prime}\right)$. As we can see, MMDT can reduce seven arcs compared with MDT, while both use only one wavelength.

\section{B. Two simulation settings and the performance metrics}

Our algorithms can work in arbitrary directed graphs, meaning that unidirectional arcs and edges corresponding to two arcs on opposite directions can coexist in the graph, and the costs for the arcs can be given differently, even with arcs on opposite directions. However, the algorithms proposed in [1] and [3] supposed to work with bidirected graph, in which all the links are all bidirectional. Thus, for a fair comparison, we divided the simulations into two settings. In the first setting, all the algorithms are run on bidirected graphs, and in the second one, they are run on arbitrary directed ones.

Three performance metrics are taken into account in the simulations: the number of wavelengths required, the total cost and the diameter of the resultant routes (light-trails or light-forests). The diameter is defined as the number of maximal hop counts from the source to all the destinations. The reason for evaluation of this metric is that it can be 


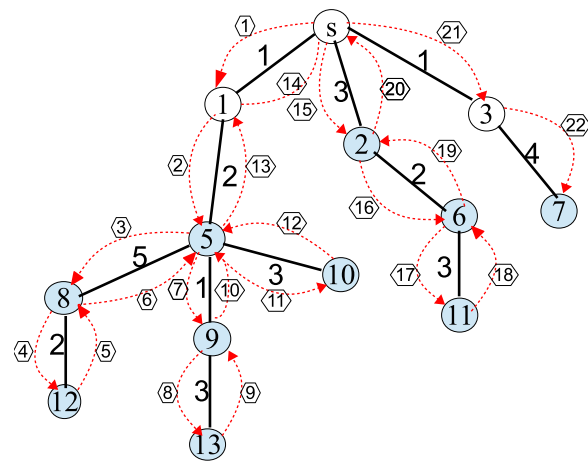

(a) The MDT

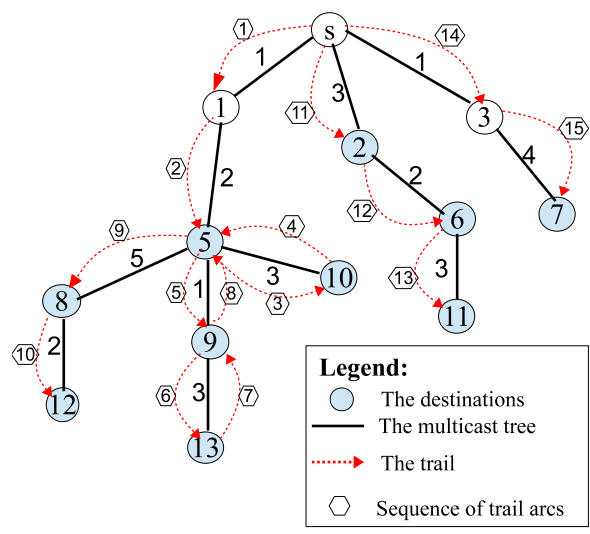

(b) The MMDT

Fig. 4: Illustration of the MDT and MMDT

represented for the end-to-end maximal delay. In fact, the delay can be combined by switching, queueing, transmission and propagation components. In all-optical networks, because of the high light speed, the propagation delay can be assumed to be the same on different links, and it is much less than the other components at the hops. Thus, the number of hops that the light signal has to pass by is usually used to represent for the delay.

\section{Experimental results with bidirected graphs}

In this setting, the considered algorithms have been run on several random bidirected graphs with different number of nodes $N=\{100,200,300\}$, the costs of arcs are randomly selected from the set of integer $\{1,2, . ., 20\}$, and the set of destinations $D$ are also randomly selected with different size $|D|=\{10,20, . ., N / 2\}$. To be sure that there is a feasible solution for all the algorithms, the selected graph must be connected and there is at least one directed path from the source to each destination for every simulation. Moreover, in order to guarantee a good confidence interval, for each size $|D|$, we run 100 simulations with different source and destination set. That means, each point in the resultant figures below is calculated on average of 100 (successful) simulations.

Besides, to respect the effect of the coefficient $\alpha$ on the performance of the proposed algorithms in [3] (FG and NG), we also set the coefficient $\alpha$ in $\{50,100,150\}$. The simulations showed that, in the cases of $\alpha=\{50,100\}$ only FG and
NG give slightly different results, in which the number of wavelengths is slightly higher and the total cost is slightly lower than those in the case of $\alpha=150$. Thus we just show the results for the case of $\alpha=150$. Likewise, we just show the results for the case of $N=\{200,300\}$ (Figures 5 and 6 ). In the case of $N=100$, the results are quantitatively the same.

In Figures 5(a) and 6(a), FG and NG result in large number of wavelengths, ranging from 1 to 5 in 200 node-networks, and from 1 to 9 in 300 node-networks when the group size varies from 10 to $N / 2$, while MDT and MMDT and the two heuristics of our algorithm draw a horizontal line with just one wavelength.

In Figures 5(b) and 6(b), the two heuristics proposed in [1] appear with the highest cost, then the two heuristics proposed in [3], the two heuristics of our algorithm have the lowest cost with a small difference between them.

In Figures 5(c) and 6(c), about the diameter, unsurprisingly, the two heuristics proposed in [1] appear with highest diameter, the two heuristics proposed in [3] achieve a constant low diameter, and the two heuristics of our algorithm are in the middle with lower diameter of NF.

Generally, in bidirectional graphs, our algorithms result in the light-trail(s) in which the number of required wavelengths is close to one, low cost but quite high diameter compared with the other algorithms.

\section{Experimental results with directed graphs}

The configurations of this setting are similar as the ones in the first setting, except that the graphs we work with are all arbitrary directed graphs. Similarly, we just show the figures for the case of $N=\{200,300\}, \alpha=150$ (Figures 7 and 8). In the other cases $(N=100, \alpha=\{50,100\})$ the results are quantitatively the same.

At first, in these configurations, MDT and MMDT almost have no solution so we do not show their results.

In Figures 7(a) and 8(a), all the algorithms result in the increasing number of wavelengths when the group size increases. FG and NG result in larger number of wavelengths with the highest of NG, while FF and NF slowly increase, with the lowest of FF. In short, FF and NF provide a lowest number of wavelengths with a slight difference between them.

In Figures 7(b) and 8(b), all the algorithms appear with the same cost but FF being a slightly better.

In Figures 7(c) and 8(c), again, FG and NG achieve a constant low diameter with a slight difference between them (lower than $25 \%$ of the number of nodes). Between the two our heuristics, NF results in lower diameter (lower than 35\% of the number of nodes). Especially, it gets closer to the FG and NG, and almost the same when the group size gets closer to $50 \%$. In contrast, FF results in high diameter (around 50\% of the number of nodes).

In short, in arbitrary directed graphs, our algorithms produce the light-trails with low number of required wavelengths, a low cost but quite high diameter compared with the other algorithms. Between the two heuristics, NF provides a good trade-off among the performance metrics. 


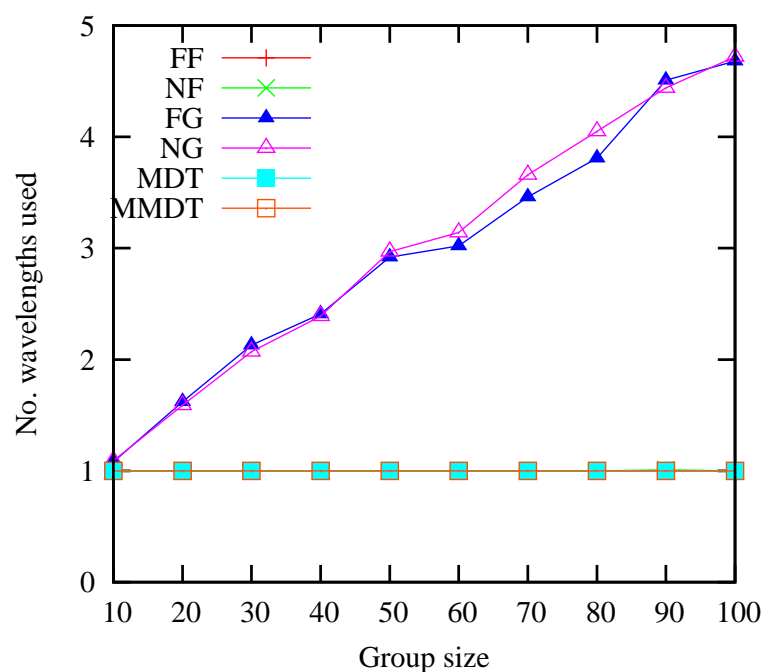

(a) No. Wavelengths vs. Group size

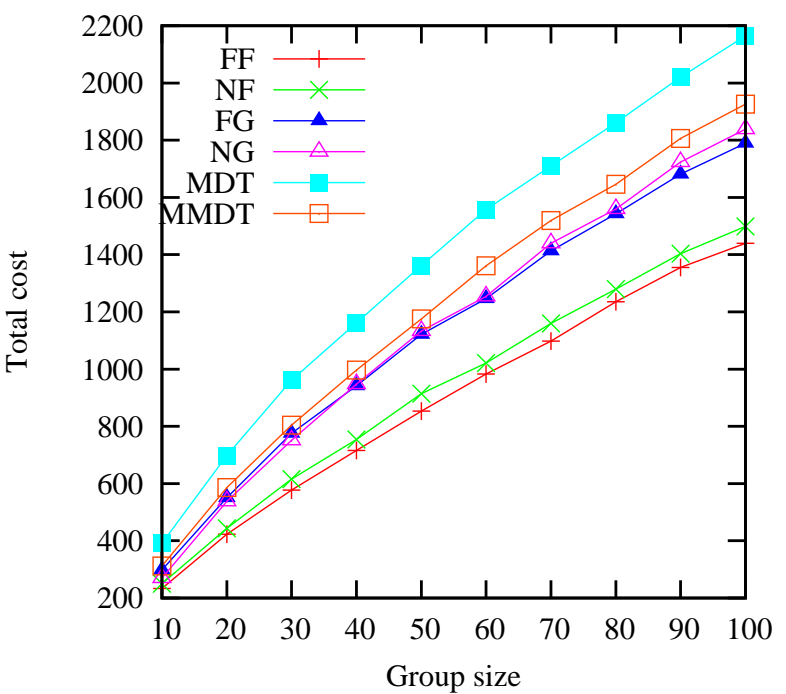

(b) Total Cost vs. Group size

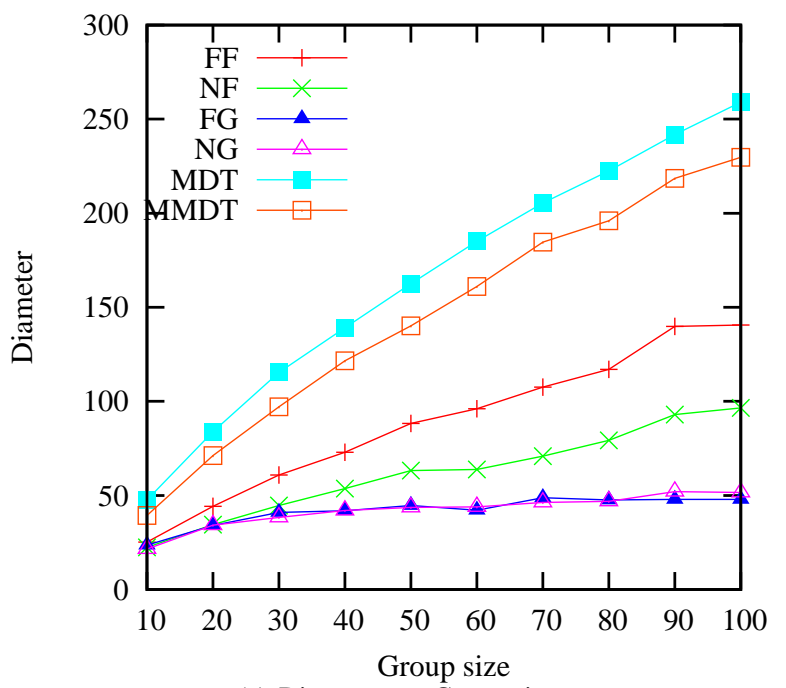

(c) Diameter vs. Group size

Fig. 5: Performance of algorithms on 200-node bigraphs

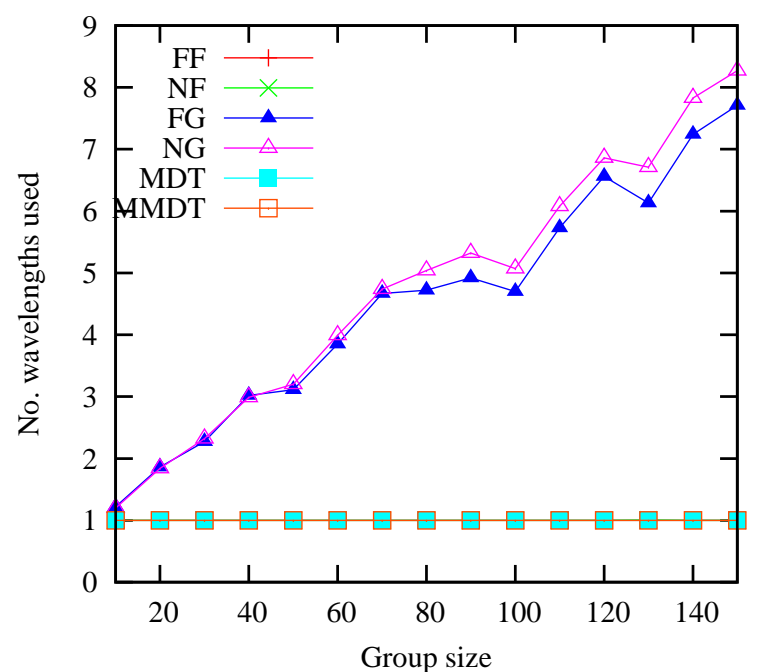

(a) No. Wavelengths vs. Group size

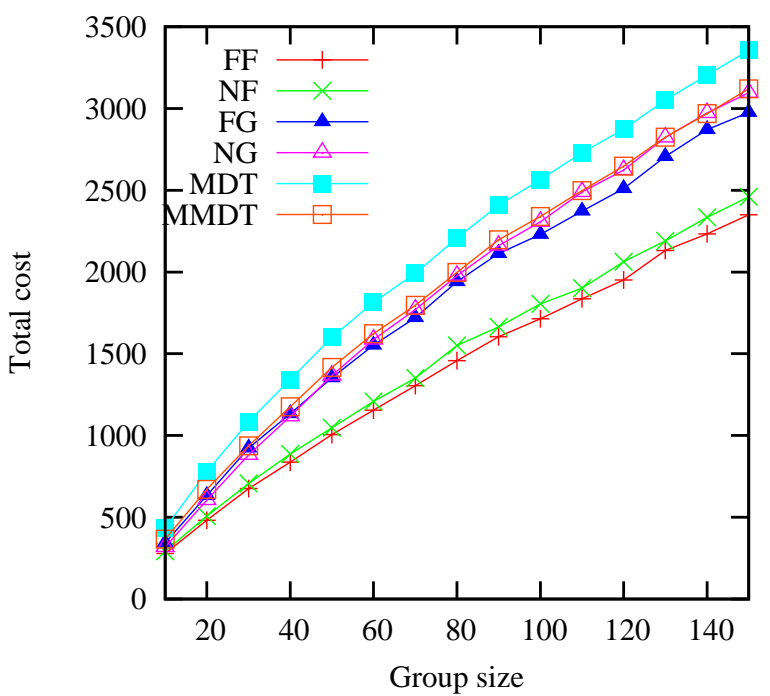

(b) Total Cost vs. Group size

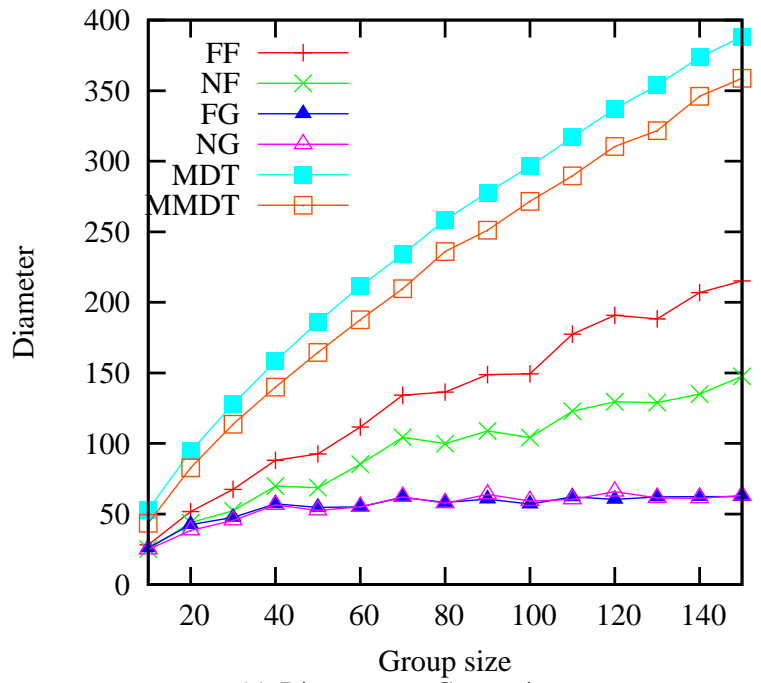

(c) Diameter vs. Group size

Fig. 6: Performance of algorithms on 300-node bigraphs 


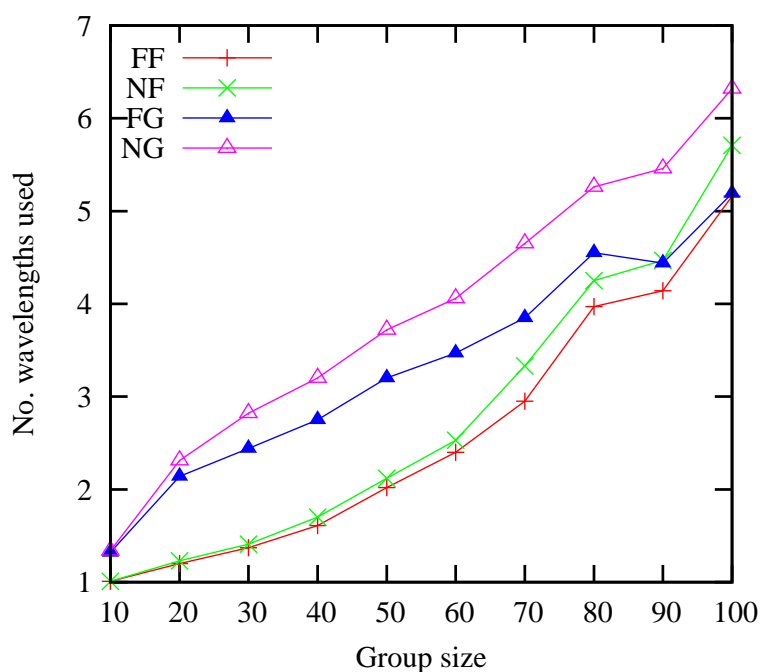

(a) No. Wavelengths vs. Group size

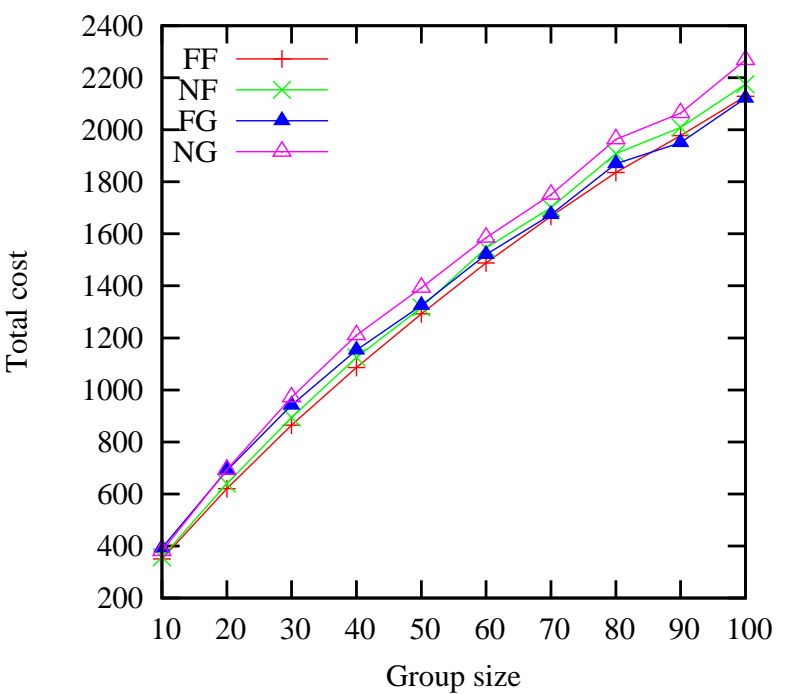

(b) Total Cost vs. Group size

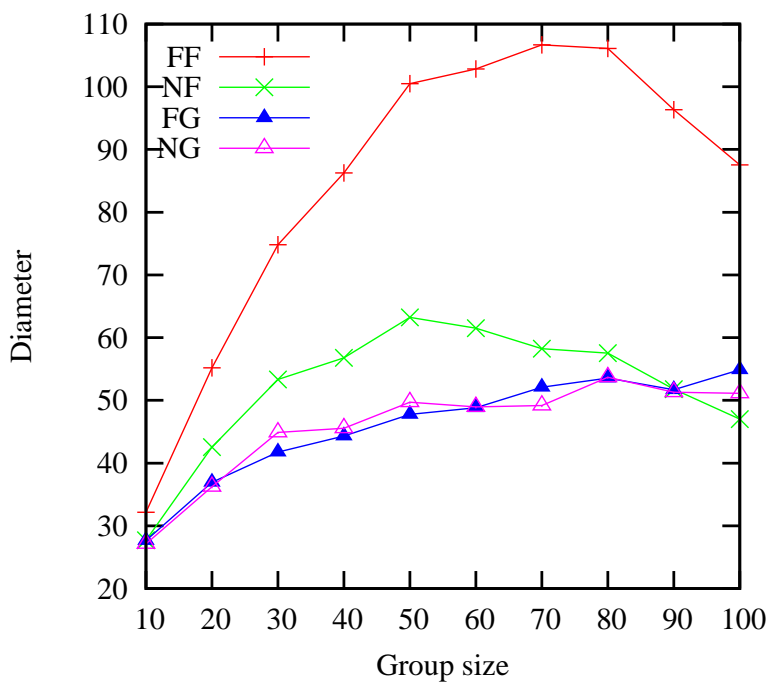

(c) Diameter vs. Group size

Fig. 7: Performance of algorithms on 200-node digraphs

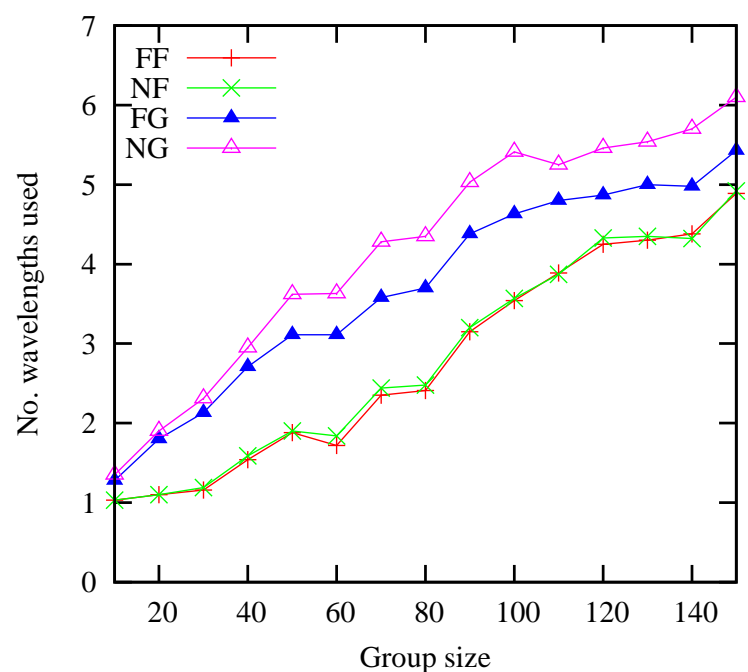

(a) No. Wavelengths vs. Group size

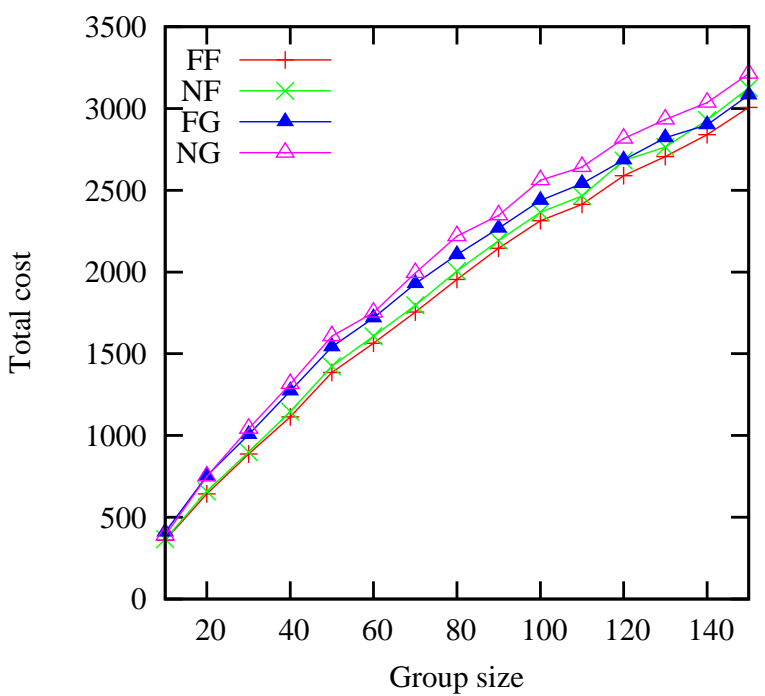

(b) Total Cost vs. Group size

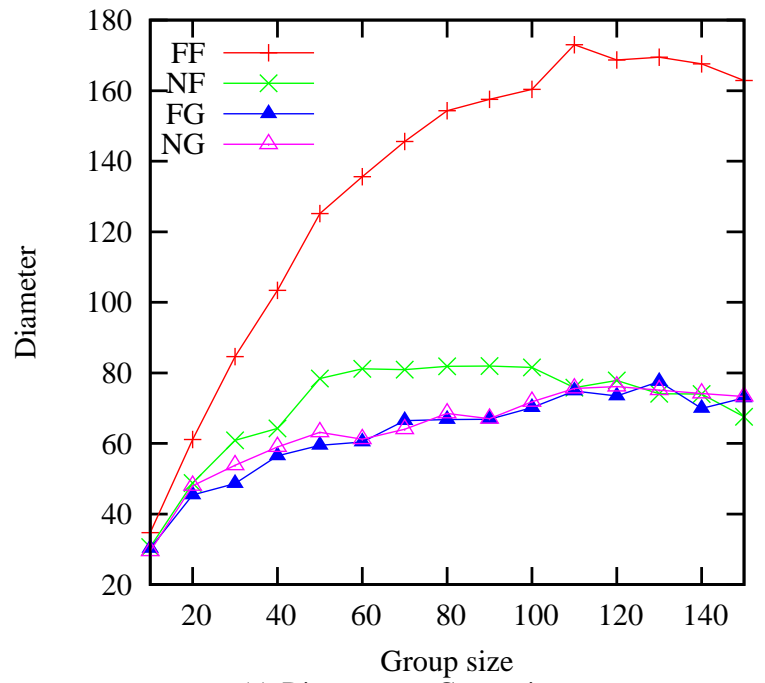

(c) Diameter vs. Group size

Fig. 8: Performance of algorithms on 300-node digraphs 


\section{E. Experimental result analysis}

\section{MDT versus MMDT}

MDT and MMDT are just suitable in symmetric networks in which they always result in optimal wavelengths but high cost and diameter. By taking advantage of multiple transmitters, MMDT also needs just one wavelength (Figures 5(a) and 6(a)) and is even better than MDT in term of cost (Figures 5(b) and 6(b)) and diameter (Figures 5(c) and 6(c)). However, both have very poor performances in arbitrary directed graphs, even have no solution in most of the cases. This is because only one arc missing on the computing trail can make MDT and MMDT fail to get a solution.

\section{Light-hierarchy based solutions versus light-tree based solutions}

As seen in the experimental results above, our lighthierarchy based solutions (FF and NF) outperform light-tree based solutions (FG and $\mathrm{NG}$ ) in term of the number of wavelengths and also the total cost, especially in bidirectional networks, with the expense of the diameter. This can be explained as follows.

The two approaches start with the same DSPT tree. In the rerouting phase, FG and NG try to extend the tree but always keep the tree structure which does not allow multiple visits at nodes. Moreover, the nodes used for the tree extension are always restricted by the source or the leaves which are either the farthest (FG) or nearest (NG) destinations in each subtree of the computed trees. These two properties limit the number of destinations to be covered in one tree, inducing larger number of wavelengths needed and higher cost. Besides, when the destinations cannot be routed in the current tree, they are routed in a new tree by the shortest paths, so the diameter is short.

In contrast, our approach is more flexible. After the first step, the route structure is no longer a tree, but a set of trails (composing a hierarchy) which allows to return some vertices more than once without conflicts. Hence, more arcs can be used for the trail extension. In addition, since all the terminals of the existing trails (and the source) can be considered, more nodes can be used for the trail extension. In short, more nodes and arcs can be used for the trail extension. This property helps to increase the number of destinations that can be covered in one trail, resulting in a fewer number of wavelengths required, but, of course, with a longer diameter. Besides, because more nodes are considered for the lowest cost, a lower total cost of the final trails can be achieved.

\section{Farthest First versus Nearest First}

As shown in the experimental results above, FF results in higher diameter but a slightly fewer wavelengths and a quasisimilar cost compared with NF. This can be explained as follows.

First, the diameter is the number of hops (the length) of the longest trail. When the selected trail (the routed trail) is replaced by an other one (the routing trail), the new routing trail must be longer (in term of cost) than the routed trail. Thus, the longer the routed trail is, the longer the new length can be. FF chooses the longest trail in the maximal clique, hence it makes the new trail longer than NF does. Furthermore, because the new trail is usually longer than routed trail in the old maximal clique, and it will probably become the farthest one in the new maximal clique and will be first considered next time. Hence it becomes longer and longer, and finally it can correspond to the diameter of the final trails. That is the reason for the fact that $\mathrm{FF}$ results in a longer diameter than NF.

Similarly, since FF tends to include more destinations in a long trail, the probability that the number of wavelengths that can be reduced by FF is higher than by NF. So FF results in a fewer wavelengths than NF does.

Finally, when the routed trail is replaced, the reduced cost is calculated by the cost of the routed trail minus the cost of the extended path of the routing trail (or the total cost of the routing trail if the source is selected). Thus, the longer the selected trail is, probably that the more the reduced cost can be. Since FF chooses the longest trail and NF chooses the shortest one in the maximal clique to diminish first, FF can reduce more cost than NF.

\section{CONCLUSION}

In this paper we address the multicasting problem in alloptical networks without splitters. The problem is to find a set of light-trails which minimizes the number of required wavelengths with a low cost. The problem is proved to be NP-hard, and two heuristics based on Shortest Path Tree are proposed to make a feasible solution, Farthest First and Nearest First. The idea of our algorithm is to diminish the conflict between the light-trails until it cannot be reduced. Especially, unlike the common approaches which assume to work on networks with bidirectional links, our algorithm can work well in arbitrary networks.

The two heuristics of our algorithm are compared with the proposed algorithms in the literature, and the simulation results showed that our algorithm achieves low number of used wavelengths, low cost but quite high diameter. Between the two, although the Farthest First can result in smaller number of wavelengths and lower cost, the Nearest First provides a better trade-off among the three performance metrics.

\section{REFERENCES}

[1] M. Ali and J. S Deogun. Cost-effective implementation of multicasting in wavelength-routed networks. IEEE/OSA Journal of Lightwave Technology, 18:1628-1638, 2000.

[2] M. Ali and J. S Deogun. Power-efficient design of multicast wavelength routed networks. IEEE Journal on Selected Areas in Communications, 18:1852-1862, 2000.

[3] Der-Rong Din. Heuristic Algorithms for Finding Light-Forest of Multicast Routing on WDM Network. Information Science and Engineering, 25:83-103, 2009.

[4] Dinh Danh Le, Miklos Molnar, and Jerome Palaysi. All-Optical Multicast Routing Algorithms without Splitters. Technical report, May 2013.

[5] Deying Li, Xiufeng Du, Xiaodong Hu, Lu Ruan, and Xiaohua Jia. Minimizing number of wavelengths in multicast routing trees in WDM networks. Networks, 35(4):260-265, 2000.

[6] Miklos Molnar. Hierarchies to Solve Constrained Connected Spanning Problems. Technical Report 11029, LIRMM, September 2011.

[7] H. Takahashi and A. Matsuyama. An approximate solution for the Steiner problem in graphs. Mathematica Japonica, 24:573-577, 1980. 
[8] Yingyu Wan and Weifa Liang. On the minimum number of wavelengths in multicast trees in WDM networks. Networks, 45(1):42-48, 2005.

[9] Xijun Zhang, John Wei, and Chunming Qiao. Constrained multicast routing in WDM networks with sparse light splitting. IEEE/OSA Journal of Lightwave Technology, 18:1917-1927, 2000.

[10] Fen Zhou, Miklos Molnar, and Bernard Cousin. Light-Hierarchy: The Optimal Structure for Multicast Routing in WDM Mesh Networks. In Computers and Communications (ISCC), 2010 IEEE Symposium on The 15th IEEE Symposium on Computers and Communications (ISCC2010), 2010, pages 611 - 616, Riccione Italie, June 2010.

[11] Yinzhu Zhou and Gee-Swee Poo. Optical multicast over wavelengthrouted WDM networks: A survey. Optical Switching and Networking, 2(3): $176-197,2005$

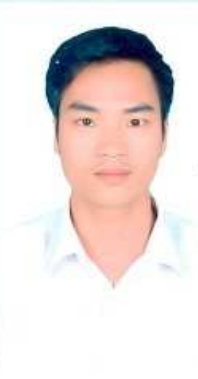

Dinh Danh Le is now a PhD student in Informatics Department at laboratory LIRMM, University Montpellier 2, France. He was graduated at the Information Technology Faculty of Hong Duc University, Vietnam in 2004 and received a master degree in Computer Science at Information Technology Faculty of Vietnam National University, Hanoi in 2007. His main research topics and interests include network evaluation, multicast routing, optical networks, and combinatorial optimization.

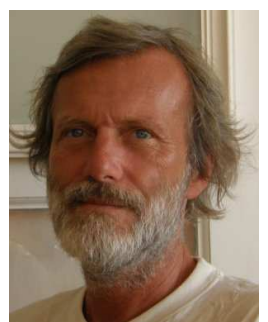

Miklós Molnár is with the University Montpellier 2, laboratory LIRMM and he is a full professor in Computer Science. He was graduated at the Faculty of Electrical Engineering, University BME of Budapest in 1976 and received the Ph.D. degree in Computer Science from the University of Rennes 1 in 1992 and the French HDR degree in 2008. His main research activity is in combinatorial optimization to solve network related problems. His results are related to NP-hard optimization problems, constrained spanning and Steiner problems, routing algorithms for unicast, in-cast and multicast communications in optical and in wireless networks, dependable communications, energy aware protocols and optimization.

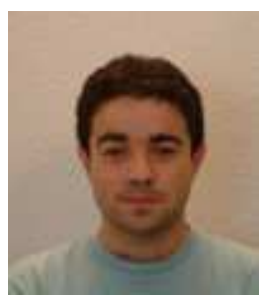

Jérôme Palaysi is an associate professor at University Montpellier 2 since September 2003. He is currently a member of the MAORE research team at LIRMM and teacher at the department of Computer Science of the IUT. He obtained his Ph.D in computer science ("Algorithmic Problems in all-optical networks") at Montpellier 2 University. During past years, he focused his research on algorithmic studies of routing problem in networks (all-optical network, wireless networks and sensor networks). He is also applying well-known methods of scheduling theory and is working with researchers in this field. 